\title{
Indicações de colonoscopia versus achado de pólipos e neoplasias colorretais
}

\section{Indications of colonoscopy versus polyps and colorectal neoplasms finding}

\author{
RODRIGO ROCHA BATISTA ${ }^{1}$, RAFAEL FERREIRA CORREIA LIMA ${ }^{1}$, \\ MARCUS FÁBIO MAGALHÃES FONSECA², LILIAN RAMOS TODINOV², GALDINO JOSÉ SITONIO FORMIGA ${ }^{3}$
}

${ }^{1}$ Médico Residente do Serviço de Coloproctologia do Hospital Heliópolis, Filiado da Sociedade Brasileira de Coloproctologia. ${ }^{2}$ Médico Assistente do Serviço de Coloproctologia do Hospital Heliópolis, Titular da Sociedade Brasileira de Coloproctologia.

${ }^{3}$ Chefe do Serviço de Coloproctologia do Hospital Heliópolis, Titular da Sociedade Brasileira de Coloproctologia.

BATISTA RR, LIMA RFC, FONSECA MFM, TODINOV LR, FORMIGA GJS. Indicações de colonoscopia versus achado de pólipos e neoplasias colorretais. Rev bras Coloproct, 2011;31(1): 64-70.

RESUMO: Objetivo: Analisar as indicações de colonoscopia e o achado de pólipos e neoplasias colorretais. Métodos: Estudo retrospectivo de laudos de colonoscopias realizadas entre janeiro de 2009 e março de 2010. As variáveis analisadas foram: idade, sexo, indicação do exame e achado de pólipo ou tumor. Realizou-se análise estatística com o teste do qui-quadrado, considerado significante quando p $<0,05$. Resultados: Foram revisados 493 laudos de colonoscopias de pacientes entre 18 e 94 anos, com média de idade de 56,7 anos, sendo $54 \%$ do sexo feminino. Os exames tiveram uma ou mais alterações em $47,3 \%$, sendo $17,4 \%$, com tumor, e $14,8 \%$, com pólipos. As colonoscopias incompletas totalizaram 24,3\%, devido ao tumor obstrutivo, à dobra fixa, ao mau preparo, à estenose e agitação psicomotora. As indicações estatisticamente significantes para maior achado de tumor colorretal foram: sangramento digestivo, anemia crônica, síndrome consuptiva, tumor abdominal palpável, polipose e elevação do antígeno cárcino-embriogênico (CEA) no pós-operatório. Não houve significância entre presença de pólipos e sexo masculino ou avanço da idade, nem entre o achado de pólipos e risco de tumor associado. Conclusão: Pacientes com sangramento digestivo, anemia crônica, síndrome consuptiva, tumor abdominal palpável, polipose e elevação de CEA no pós-operatório de câncer colorretal devem ser priorizados para o estudo colonoscópico.

Palavras-chave: indicações; colonoscopia; diagnóstico; pólipo; neoplasia.

\section{INTRODUÇÃO}

O câncer colorretal (CCR) é a terceira neoplasia mais frequente, responsável por um milhão de casos e 600.000 mortes a cada ano ${ }^{1}$.

A colonoscopia é um método adequado na investigação das doenças colorretais, com destaque para os pólipos e neoplasias, sendo considerada diagnóstica e terapêutica ${ }^{2-4}$. Permite a identificação precoce de lesões em pessoas de grupos de risco, investiga os sinais e sintomas (dor abdominal, sangramento digestivo, alteração do hábito intestinal, diarreia crônica, anemia, massas abdominais), visualiza a mucosa do íleo terminal, cólons, reto e faz a análise macroscópica das lesões encontradas. Permite também realizar procedimentos como biópsia, polipectomia, hemostasia, mucosectomia, dilatação de estenose, colocação de prótese, descompressão colônica em dilatação aguda e tatuagem de lesões para futura revisão endoscópica ou cirurgia $^{2-4}$. Além disso, permite reduzir a incidência do CCR por meio de polipectomias 5 .

No entanto, trata-se de um exame invasivo e que necessita de sedação, cuja indicação deve ser baseada em sinais, sintomas e fatores de risco individual ou familiar ${ }^{6,7}$.

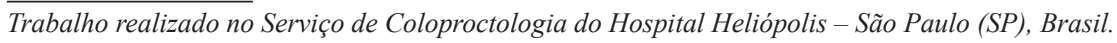

Recebido em: 06/12/2010

Aprovado em: 14/04/2011 


\section{OBJETIVO}

Analisar as indicações de colonoscopia e o achado de pólipos e neoplasias colorretais.

\section{PACIENTES E MÉTODOS}

Foi realizado um estudo retrospectivo com revisão de 493 laudos de colonoscopias realizadas em pacientes ambulatoriais e internados no Hospital Heliópolis, em São Paulo, entre janeiro de 2009 e março de 2010. As variáveis analisadas foram: idade, sexo, indicação do exame e achado de pólipo ou tumor.

Os exames foram realizados com videocolonoscópio ou fibrocolonoscópio Olympus ${ }^{\circledR}$, uso de sedação consciente com midazolam e opióide (petidina ou fentanil) intravenoso e preparo do cólon anterógrado constituído de dieta sem resíduos, bisacodil e manitol 10\%. Os pacientes portadores de ostomias (ileostomia ou colostomia, derivativa ou terminal) tiveram preparo complementado com enteroclisma de clister glicerinado $12 \%$.

O exame colonoscópico foi considerado completo quando atingiu o ceco ou íleo terminal. Foram excluídos os pacientes com idade inferior a 18 anos e aqueles submetidos à cirurgia colorretal em outro serviço.

As indicações de colonoscopias foram obtidas com base em informação contida nos laudos de solicitação do exame. Cada laudo de exame contém apenas uma indicação de colonoscopia, em geral, a mais relevante no momento em que foi solicitada.

Optou-se por agrupar, como "sangramento digestivo", as seguintes indicações de colonoscopias: sangue oculto positivo, enterorragia, hemorragia digestiva alta e baixa e sangramento retal. E por agrupar, como "doença inflamatória intestinal (DII)", as indicações doença de Crohn (DC), retocolite ulcerativa (RCU), retite inespecífica e pós-operatório de DC ou RCU. A indicação "seguimento oncológico" corresponde aos pacientes em pós-operatório de cirurgia por CCR que fizeram o exame de rotina, enquanto a indicação "elevação do antígeno cárcino-embriogênico (CEA)" diz respeito aos pacientes em pós-operatório de cirurgia por CCR que apresentaram suspeita de recidiva da doença. Quanto às demais indicações de colonoscopia, aquelas que individualmente corresponderam a menos de $0,5 \%$ do total da amostra foram agrupadas como "outras indicações".

A análise estatística foi feita com o teste do quiquadrado de Pearson, considerado significante quando $\mathrm{p}<0,05$.

\section{RESULTADOS}

Dos 493 laudos de colonoscopias incluídos no estudo, $54 \%$ eram de pacientes do sexo feminino e $46 \%$ do masculino, com idade entre 18 e 94 anos e média de idade de 56,72 anos (Figura 1).

As indicações de colonoscopia nesta amostra foram: seguimento oncológico (15,4\%), sangramento digestivo (15\%), pesquisa de tumor sincrônico $(12,6 \%)$, DII $(11,4 \%)$, rastreamento de CCR $(6,3 \%)$, diarreia crônica $(4,1 \%)$, constipação crônica $(4,1 \%)$, nódulo(s) hepático, pulmonar e cervical (3,9\%), dor abdominal (3,9\%), controle pós-polipectomia (3,2\%), síndrome consuptiva (3\%), alteração do hábito intestinal $(2,8 \%)$, doenças orificiais $(2,8 \%)$, massa abdominal palpável (2\%), pólipo ao exame proctológico $(1,6 \%)$, anemia crônica $(1,4 \%)$, polipose $(1,2 \%)$, história familiar de polipose $(0,8 \%)$ e elevação de CEA no pós-operatório de CCR $(0,6 \%)$. Outras indicações somaram 3,9\% do total (Tabela 1).
A

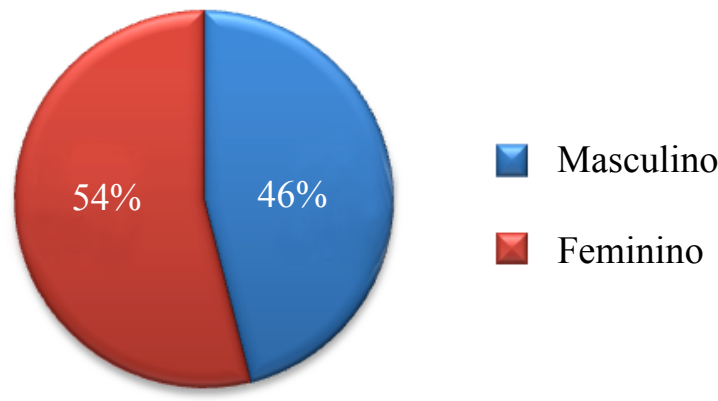

B

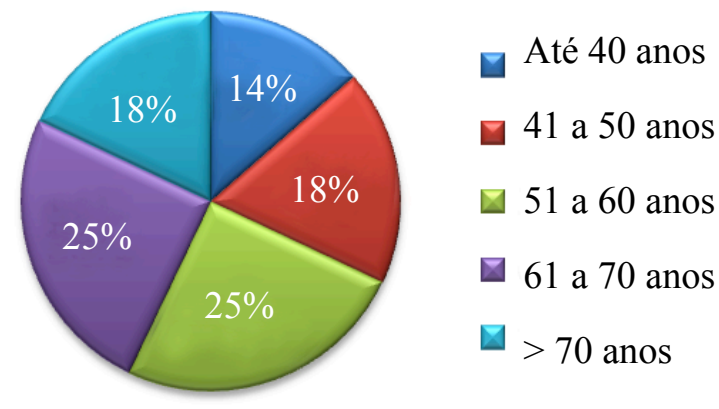

Figura 1. A: distribuição da amostra por sexo; B: distribuição da amostra por faixa etária. 
Os exames foram normais em $52,7 \%$ dos pacientes, enquanto $47,3 \%$ tiveram uma ou mais alterações. Os achados significativos em relação ao total de exames foram: tumoração colorretal $(17,4 \%)$, diverticulose $(15,6 \%)$, pólipos $(14,8 \%)$, úlceras $(3,9 \%)$ e angiodisplasia $(0,4 \%)$ (Figura 2). Com relação à localização dos pólipos e tumores encontrados, $77,91 \%$ dos tumores e $67,18 \%$ dos pólipos estavam no reto e cólon esquerdo (Figura 3).

O exame colonoscópico foi completo em $75,7 \%$, enquanto $24,3 \%$ dos exames não progrediram até ceco ou íleo terminal. A distribuição, conforme topografia alcançada, foi: íleo terminal $(18,1 \%)$, ceco $(57,6 \%)$, ascendente $(2,2 \%)$, ângulo hepático $(4,7 \%)$, transverso $(2,6 \%)$, ângulo esplênico (3\%), descendente $(2,4 \%)$, sigmoide $(4,1 \%)$, transição retossigmoide $(2,1 \%)$ e reto $(3,2 \%)$. As causas da não-progressão do exame foram: tumor obstrutivo $(29,2 \%)$, dobra fixa $(26,7 \%)$, mau preparo $(17,5 \%)$, estenose (inflamatória, diverticular, actínica, anastomótica $-9,2 \%)$, agitação psicomotora $(5,8 \%)$, dor $(4,2 \%)$, compressão extrínseca $(3,3 \%)$, dolicossigmoide $(2,5 \%)$, intussuscepção $(0,8 \%)$ e aderência cirúrgica $(0,8 \%)$.

$\mathrm{O}$ anatomopatológico da biópsia dos tumores mostrou adenocarcinoma em 94,2\% dos casos; linfoma não-Hodgkin, em 2,3\%; processo inflamatório crônico granulomatoso, em 1,2\%; e colite crônica inespecífica, em 2,3\% (Figura 4). Quanto à forma dos

Tabela 1. Distribuição das indicações de colonoscopia em percentagem do total de exames.

\begin{tabular}{llll}
\hline Indicação & $\mathbf{\%}$ & Indicação & \% \\
\hline Seguimento oncológico & 15,4 & Síndrome consuptiva & 3 \\
Sangramento digestivo & 15 & Alteração do hábito intestinal & 2,8 \\
Pesquisa de tumor sincrônico & 12,6 & Doença orificial & 2,8 \\
DII & 11,4 & Massa abdominal & 2 \\
Rastreamento de CCR & 6,3 & Pólipo ao exame proctológico & 1,6 \\
Diarreia crônica & 4,1 & Anemia crônica & 1,4 \\
Constipação crônica & 4,1 & Polipose & 1,2 \\
Nódulo(s) (hep, pulm, cerv) & 3,9 & História familiar de polipose & 0,8 \\
Dor abdominal & 3,9 & Elevação de CEA & 0,6 \\
Controle pós-polipectomia & 3,2 & Outras indicações & 3,9 \\
\hline
\end{tabular}

A

Normais

Alteradas

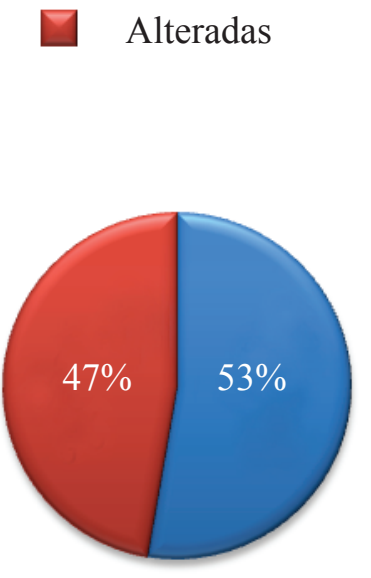

B

\% dos pacientes

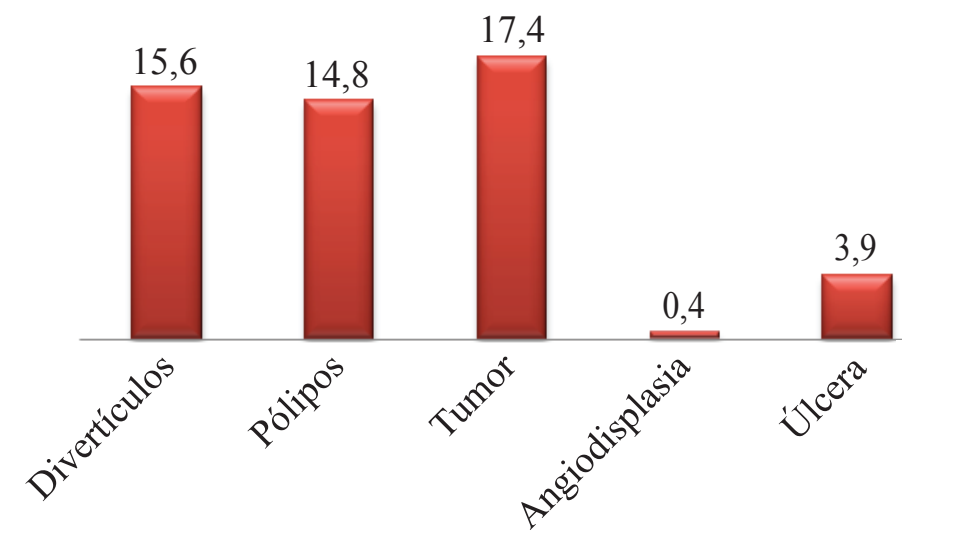

Figura 2. A: distribuição das colonoscopias normais e alteradas; B: achados significativos em percentagem do total de exames. 


\section{Distribuição dos pólipos e tumores}

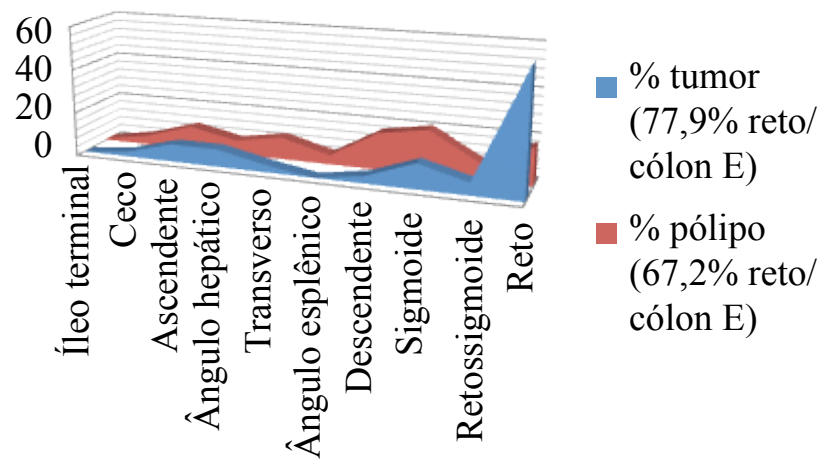

Figura 3. Distribuição dos tumores (em azul) e pólipos (em vermelho), desde íleo terminal até o reto. Destaca-se a predominância dos pólipos e tumores no reto, sigmoide e descendente.

\section{Anatomopatológico dos tumores (biópsia)}

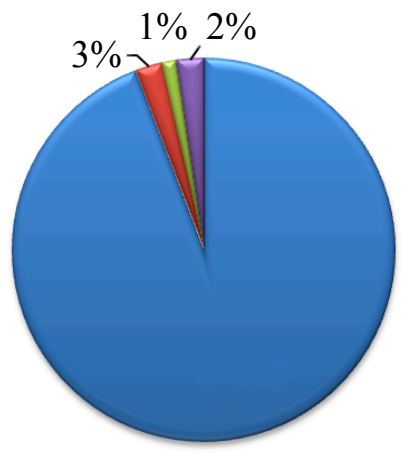

Adenocarcinoma

Lindoma não-Hodgkin

Processo inflamatório crônica granulomatoso

Coleto crônica inespecífica

Figura 4. Resultado anatomopatológico dos tumores (biópsia) em percentagem do total de exames.

\section{Anátomopatológico dos pólipos (biopsia ou polipectomia}

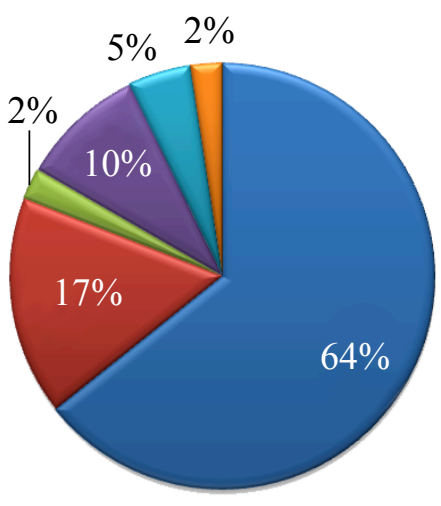

Adenoma

Adenocarcioma in situ

Adenocarcinoma invasivo

- Hiperplásico

Juvenil

Inflamatório

Figura 5. Resultado anatomopatológico dos pólipos (biópsia ou polipectomia) em percentagem do total de exames.

pólipos, $58 \%$ eram sésseis, $27 \%$ pediculados e $15 \%$ subpediculados. O anatomopatológico dos pólipos foi: adenoma (64\%), adenocarcinoma in situ (17\%), hiperplásico (10\%), juvenil (5\%), adenocarcinoma invasivo (2\%) e inflamatório (2\%) (Figura 5).

As indicações com achado de tumoração colorretal foram: elevação de CEA no pós-operatório de CCR $(66,7 \%)$, polipose $(33,3 \%)$, massa abdominal palpável (30\%), anemia crônica $(28,6 \%)$, sangramento digestivo $(13,5 \%)$, síndrome consuptiva $(13,3 \%)$, dor abdominal $(5,3 \%)$, pesquisa de tumor sincrônico $(3,2 \%)$ e seguimento oncológico $(2,6 \%)$. Os exames realizados para as demais indicações não encontraram lesões sugestivas de neoplasia colorretal (Tabela 2). Quando as

Tabela 2. Percentagem dos exames positivos para o achado de tumor colorretal, de acordo com cada indicação.

\begin{tabular}{llll}
\hline Indicação versus tumor & $\mathbf{\%}$ & Indicação versus tumor & $\mathbf{\%}$ \\
\hline Elevação de CEA & 66,7 & Diarreia crônica & 0 \\
Polipose & 33,3 & Constipação crônica & 0 \\
Massa abdominal & 30 & Alteração do hábito intestinal & 0 \\
Anemia crônica & 28,6 & Rastreamento & 0 \\
Sangramento digestivo & 13,5 & Pólipo ao exame proctológico & 0 \\
Síndrome consuptiva & 13,3 & DII & 0 \\
Dor abdominal & 5,3 & História familiar de polipose & 0 \\
Pesquisa de tumor sincrônico & 3,2 & Doença orificial & 0 \\
Seguimento oncológico & 2,6 & Nódulo(s) (hep, pulm, cerv) & 0 \\
Controle pós-polipectomia & 0 & Outras indicações & 0
\end{tabular}


indicações com achado superior a $10 \%$ de tumor foram comparadas com as demais, foi encontrada diferença estatisticamente significante (Tabela 3 ).

Quando tentou-se relacionar presença ou não de pólipos com sexo e idade, não foi encontrada diferença significante entre os grupos (Tabela 4). A média de idade entre os pacientes portadores de pólipos era de 60 anos, enquanto os pacientes sem pólipos tinham média de 56 anos $(\mathrm{p}>0,05)$. Além disso, não houve significância entre o achado de pólipos e o risco de tumor associado (Tabela 5), nem entre a frequência de pólipos e tumores encontrados nos exames de seguimento oncológico e demais indicações (Tabelas 6 e 7).

Tabela 3. Comparação entre o grupo de indicações com mais de 10\% de tumor versus grupo de indicações com menos de $10 \%$ de tumor, com diferença estatisticamente significante $(p<0,05)$. Foram excluídos da análise os pacientes sabidamente portadores de neoplasia colorretal com indicação de pesquisa de lesão sincrônica.

\begin{tabular}{|c|c|c|c|c|}
\hline \multirow{2}{*}{ Indicação } & & \multicolumn{2}{|c|}{ Tumor } & \multirow{2}{*}{ Total } \\
\hline & & Sim & Não & \\
\hline \multirow[t]{2}{*}{ Indicação com } & $>10 \%$ tumor & 21 & 94 & 115 \\
\hline & $<10 \%$ tumor & 3 & 313 & 316 \\
\hline Total & & 24 & 407 & 431 \\
\hline
\end{tabular}

Tabela 4. Comparação entre sexo versus presença ou não de pólipos, sem diferença estatisticamente significante $(p=0,104)$.

\begin{tabular}{lccc}
\hline \multirow{2}{*}{ Sexo } & \multicolumn{2}{c}{ Pólipos } & \multirow{2}{*}{ Total } \\
\cline { 2 - 3 } & Sim & Não & \\
\hline Masculino & 40 & 187 & 227 \\
Feminino & 33 & 233 & 266 \\
Total & 73 & 420 & 493 \\
\hline
\end{tabular}

Tabela 5. Comparação entre o grupo de indicações com mais de $10 \%$ de pólipos versus grupo de indicações com menos de $10 \%$ de pólipos em relação ao achado de tumor colorretal, sem diferença estatisticamente significante $(p=0,685)$. Foram excluídos da análise os pacientes sabidamente portadores de neoplasia colorretal com indicação de pesquisa de lesão sincrônica.

\begin{tabular}{|c|c|c|c|c|}
\hline \multirow{2}{*}{ Indicação } & & \multicolumn{2}{|c|}{ Tumor } & \multirow{2}{*}{ Tota } \\
\hline & & Sim & Não & \\
\hline \multirow[t]{2}{*}{ Indicação com } & $>10 \%$ pólipo & 17 & 272 & 289 \\
\hline & $<10 \%$ pólipo & 7 & 135 & 142 \\
\hline Total & & 24 & 407 & 431 \\
\hline
\end{tabular}

Tabela 6. Comparação entre os exames de seguimento oncológico versus demais indicações em relação ao achado de tumor colorretal, sem diferença estatisticamente significante $(p=0,218)$. Foram excluídos da análise os pacientes sabidamente portadores de neoplasia colorretal com indicação de pesquisa de lesão sincrônica.

\begin{tabular}{lcccc}
\hline \multirow{2}{*}{ Seguimento } & & \multicolumn{2}{c}{ Tumor } & \multirow{2}{*}{ Total } \\
\cline { 3 - 4 } & & Sim & Não & \\
\hline $\begin{array}{l}\text { Seguimento } \\
\text { oncológico }\end{array}$ & Não & 22 & 333 & 355 \\
& Sim & 2 & 74 & 76 \\
Total & & 24 & 407 & 431 \\
\hline
\end{tabular}

Tabela 7. Comparação entre os exames de seguimento oncológico versus demais indicações em relação ao achado pólipos, sem diferença estatisticamente significante $(p=0,315)$. Foram excluídos da análise os pacientes sabidamente portadores de polipose colônica e pólipo ao exame proctológico.

\begin{tabular}{lcccc}
\hline \multirow{2}{*}{ Seguimento } & & \multicolumn{2}{c}{ Pólipo } & \multirow{2}{*}{ Total } \\
\cline { 3 - 4 } & & Sim & Não & \\
\hline Seguimento & Não & 47 & 356 & 403 \\
oncológico & & & & \\
& Sim & 12 & 64 & 76 \\
Total & & 59 & 420 & 479 \\
\hline
\end{tabular}

\section{DISCUSSÃO}

A maioria dos pacientes que realizaram colonoscopia no estudo é mulher (54\% do total), dado semelhante ao encontrado na literatura ${ }^{8}$. Além disso, destaca-se o achado de $47,3 \%$ de exames alterados, provavelmente devido às características do Serviço de Coloproctologia do Hospital Heliópolis (terciário e com pacientes com queixas de longa duração).

As principais indicações de colonoscopia na presente amostra foram seguimento oncológico, sangramento digestivo, pesquisa de lesão sincrônica e doença inflamatória intestinal, as quais são achados distintos em relação à literatura, que destaca como principais indicações o sangramento digestivo, a anemia crônica e a alteração do hábito intestinal ${ }^{8}$. Outras indicações comuns de colonoscopia que a literatura destaca são: diarreia crônica, doença inflamatória intestinal, pesquisa de tumor sincrônico, seguimento oncológico e de pólipos ${ }^{9}$. Esta diferença provavelmente ocorreu devido ao perfil dos pacientes admitidos no serviço mencionado. 
A taxa de chegada ao ceco ou íleo terminal neste estudo foi de $75,7 \%$, o que é semelhante a um grande estudo no Reino Unido (77,1\%), que também analisou todas as indicações e perfis de pacientes ${ }^{4}$. Outros estudos que excluíram fatores de complicação (mau preparo, lesões obstrutivas) conseguiram, desse modo, um índice de exame completo de até $98 \%{ }^{10}$. Os fatores de maior dificuldade na realização da colonoscopia são: biótipo longilíneo, mulheres (pelve larga e maior comprimento dos cólons), idosos (maior risco de depressão respiratória), lesões obstrutivas, estenoses, aderências pós-operatórias, moléstia diverticular, mau preparo e comorbidades ${ }^{4,10,11}$.

O índice diagnóstico de uma indicação de colonoscopia é a relação entre os achados significativos e o número de colonoscopias solicitadas para cada indicação. Siddique et al. mostraram que as colonoscopias com indicação apropriada possuem índice diagnóstico de 40 a $45 \%$, enquanto as com indicação inapropriada apresentam índice diagnóstico de apenas 15 a $20 \%{ }^{12}$. Assim, sintomas abdominais vagos sem indícios de sangramento, incontinência e constipação crônica são indicações com baixo índice diagnóstico ${ }^{6,7}$.

Os fatores preditores para o achado de pólipos e neoplasias colorretais são diferentes. O adenoma está asso- ciado com o avanço da idade e sexo masculino, enquanto que o CCR está relacionado à indicação do exame ${ }^{6}$. Alguns estudos mostraram 14,5\% de achado de neoplasia colorretal em pacientes com colonoscopias solicitadas devido ao sangramento digestivo, $2,2 \%$ na pesquisa de tumor sincrônico, $0,5 \%$ no controle pós-polipectomia e $0,7 \%$ nos exames de rastreamento ${ }^{6,13}$. Estes dados são semelhantes aos encontrados na presente amostra, exceto em relação aos pólipos, que não apresentaram correlação significante com sexo e avanço da idade, provavelmente devido ao número de exames analisados.

\section{CONCLUSÃO}

Pacientes com sangramento digestivo, anemia crônica, síndrome consuptiva, tumor abdominal palpável, polipose e elevação de CEA no pós-operatório de CCR devem ser priorizados para o estudo colonoscópico, visto que estas indicações estão associadas ao maior achado de neoplasias colorretais.

A média de idade dos pacientes com pólipos foi pouco superior aos pacientes sem pólipos, porém sem significância estatística. Também não houve diferença significante entre o gênero e o achado de pólipos na presente amostra.

\begin{abstract}
Objective: To analyze colonoscopy indications and the finding of polyps and colorectal neoplasms. Methods: A retrospective study of colonoscopies reports that were performed between January, 2009 and March, 2010. The analyzed variables included: age, gender, indication for examination, and found of polyp or tumor. The statistical analysis was accomplished with the chi-square test, with significance at $\mathbf{p}<\mathbf{0 . 0 5}$. Results: Four hundred and ninety-three colonoscopy reports of patients between 18 and 94 years, mean age 56.7 years old, and $54 \%$ female, were reviewed. The examinations had one or more changes in $\mathbf{4 7 . 3 \%}, \mathbf{1 7 . 4 \%}$ with tumor and $14.8 \%$ with polyps. The incomplete colonoscopies corresponded to $24.3 \%$, due to tumor obstruction, fixed kink, poor preparation, stenosis, and agitation. The statistically significant indications for increased finding of colorectal tumor were gastrointestinal bleeding, anemia, chronic wasting syndrome, palpable abdominal tumor, polyposis, and elevation of CEA in the postoperative. There were no significant differences between polyps and males or advancing age, neither between the finding of polyps and risk of associated tumor. Conclusion: Patients with gastrointestinal bleeding, anemia, chronic wasting syndrome, palpable abdominal tumor, polyposis, and increased postoperative CEA of colorectal cancer should be prioritized for the colonoscopy study.
\end{abstract}

Keywords: indications; colonoscopy; diagnosis; polyp; neoplasm.

\section{REFERÊNCIAS}

1. Atkin WS, Edwards R, Kralj-Hans I, Wooldrage K, Hart AR, Northover JMA, et al. Once-only flexible sigmoidoscopy screening in prevention of colorectal cancer: a multicenter randomized controlled trial. Lancet 2010;375:1624-33.

2. Nossa FLC, Paula Nunes BLBB, Todinov LR, Barreto Neto PF, Silva JH, Formiga GJS. Colonoscopia diagnóstica e terapêutica. Avaliação das indicações e resultados. Rev Bras
Coloproct 1999;19:168-71.

3. Formiga FB, Rocha KG, Magri KD, Carvalho MP, Credidio AV, Cruz SHA, et al. Colonoscopia: morbidade negligenciada. Rev Bras Coloproct 2009;29:345-50.

4. Nahas SC, Marques CFS, Araújo SA, Aisaka AA, Nahas CSR, Pinto RA, et al. Colonoscopia como método diagnóstico e terapêutico das moléstias do intestino grosso: análise de 2567 exames. Arq Gastroenterol 2005;42:77-82.

5. Jatobá MP, Candelária PAP, Klug WA, Fang CB, Capelhuchnik 
P. Pesquisa de sangue oculto nas fezes e achado colonoscópico em 60 pacientes. Rev Bras Coloproct 2008;28:425-30.

6. Rex DK. Colonoscopy: a review of its yield for cancers and adenomas by indication. Am J Gastroenterol 1995;90:353-65.

7. Berkowitz I, Kaplan M. Indications for colonoscopy. S Afr Med J 1993;83:245-8.

8. Santos CHM, Cury MS, Saad FT. Principais achados de colonoscopias realizadas em caráter de urgência e eletivas. Rev Bras Coloproct 2009;29:83-7.

9. Manzione CR, Nadal SR, Manzione TS, Ito FR. Indicações e achados das colonoscopias nos doentes HIV-positivo. Comparação com soronegativos. Rev Bras Coloproct 2007;27:26-30.

10. Anderson JC, Gonzalez JD, Messina CR, Pollack BJ. Factors that predict incomplete colonoscopy: thinner is not always better. Am J Gastroenterol 2000;95:2784-7.
11. Dafnis G, Granath F, Pahlman L, Ekbom A, Blomqvist P. Patient factors influencing the completion rate in colonoscopy. Digest Liver Dis 2005;37:113-8.

12. Siddique I, Mohan K, Hasan F, Memon A, Patty I, Al-Nakib B. Appropriateness of indication and diagnostic yield of colonoscopy: first report based on the 2000 guidelines of the American Society for Gastrointestinal Endoscopy. World J Gastroenterol 2005;11:7007-13.

13. Bond JH. Rectal bleeding: is it always an indication for colonoscopy? Am J Gastroenterol 2002;97:223-5.

\section{Endereço para correspondência:}

GALDINO JOSÉ SITONIO FORMIGA

Serviço de Coloproctologia do Hospital Heliópolis

Rua Cônego Xavier, 276 - Vila Nova Heliópolis

CEP 04231-030 - São Paulo (SP), Brasil

E-mail: gformiga@ig.com.br 